\title{
Feature Linking using Synchronized Responses in Chaotic Cellar Neural Networks for Visual Stimulus of Moving Objects
}

\author{
Akihiro Yamaguchi *, Satoshi Arakane \\ Department of Information and Systems Engineering, Fukuoka Institute of Technology, \\ 3-30-1 Wajiro-higashi, Higashi-ku, Fukuoka, 811-0116, JAPAN \\ Masao Kubo \\ Department of Computer Science, National Defense Academy of Japan, \\ 1-10-20 Hashirimizu, Yokosuka, Kanagawa, 239-8686, JAPAN \\ E-mail:*aki@fit.ac.jp
}

\begin{abstract}
A feature-linking mechanism using the synchronized responses of neural assemblies was studied for chaotic cellar neural networks (Chaotic-CNN). Chaotic-CNNs consist of chaotic spike response neurons that show chaotic interspike-interval dynamics. In our scheme of feature linking, the features of the target objects are linked by the synchronized spike responses characterized by the temporal chaotic pattern of the spike sequence. In this paper, we analyze the synchronized spike responses invoked by the visual stimulus of moving bars. Consequently, the resulting neural assemblies have higher correlation for the visual stimulus of two bars moving in the same direction than in the opposite direction. We also discuss the possibility of feature linking using the chaotic synchronized responses.
\end{abstract}

Keywords: chaotic synchronization, neural coding, spike response model, feature linking

\section{Introduction}

In the brain system, feature linking is one of the principal functions used to realize recognition of visual objects. From physiological experiments, correlated firing among neurons is regarded as a candidate mechanism for such feature linking ${ }^{1,2}$. In neural coding schemes that use the synchronization of neurons, feature-linking information is represented by synchronized firing among the corresponding neurons such that the features detected by neurons are linked if their firing patterns are synchronized.

On the other hand, chaotic systems are well-known for their complex behavior. Comparing with a periodic spike pattern, a chaotic spike pattern is expected to have an advantage in terms of the variety of represented information. Therefore, there is a possibility of increases the performance of information representation by using chaotic synchronization of spike responses ${ }^{3,4}$.

From the view point of neural coding, the authors have studied the formation of chaotic cell assemblies in chaotic cellular neural networks (Chaotic-CNNs), which are two-dimensional coupled networks of chaotic neurons ${ }^{5-7}$. Chaotic neurons are modeled by the chaotic spike response model (Chaotic-SRM), which is an extended spike response model (SRM), to show chaotic inter-spike intervals by adding background sinusoidal oscillation $^{5-7}$.

In our previous study, chaotic cell assemblies were formed in Chaotic-CNNs for visual stimuli when a stationary image was inputted ${ }^{6,7}$. In this research, we analyze the formation of chaotic cell assemblies for the visual stimulus of moving objects and their featurelinking property. 


\section{Chaotic Cellular Neural Networks}

Chaotic-CNNs are defined as two-dimensional coupled systems of Chaotic-SRM ${ }^{5-7}$. In the following section, the definitions of the neuron model and the network are described, respectively.

\subsection{Neuron Model}

As a neuron model, we use a Chaotic-SRM, which is an extended spike response model (SRM). The SRM was introduced by Gerstner and Kistler ${ }^{8}$. The definition of a Chaotic-SRM is as follows.

The membrane potential $u(t)$ of the neuron at time $t$ is defined as

$$
u(t)=u_{\text {rest }}+\eta\left(t-t^{*}\right)+\beta,
$$

where $u_{\text {rest }}, t^{*}$, and $\beta$ denote the resting potential, the last firing time, and the external input, respectively. The kernel function $\eta$ is defined as

$$
\eta\left(t-t^{*}\right)=-\eta_{\text {init }} \exp \left(\frac{t-t^{*}}{\tau_{\eta_{0}}}\right) \Theta\left(t-t^{*}\right),
$$

where $\tau_{\eta_{0}}$ is the time constant of the spike response and $\Theta$ is a step function such that $\Theta(s)$ is 1 for $s \geq 0$ and 0 for other values.

In this model, when the membrane potential exceeds the threshold value $\theta$, the neuron fires and the membrane potential is reset by the update of the last firing time $t^{*}$. The term $-\eta_{\text {init }}$ is the initial value of the kernel function $\eta$ after firing.

We extended the original SRM to show the chaotic response by adding a background sinusoidal oscillation in the same way as for a bifurcating neuron ${ }^{9}$ and a chaotic pulse-coupled neural network ${ }^{4}$. In our model, background oscillation is added to the term $\eta_{\text {init }}$ such that

$$
\eta_{\text {init }}=\eta_{0}-A_{\eta_{0}} \sin \left(2 \pi \omega_{\eta_{0}} t^{*}+\phi\right),
$$

where $A_{\eta_{0}}, \omega_{\eta_{0}}$, and $\phi$ denote the amplitude, the frequency, and the phase shift of the background oscillation, and $\eta_{0}$ is constant. The Chaotic-SRM shows various chaotic behaviors depending on the parameters $A_{\eta_{0}}$ and $\beta$.

\subsection{Definition of a Network}

In a Chaotic-CNN, each neuron is located in an $N \times M$ lattice and connected to neighbors. Let $n_{x, y}$ be a neuron located at the position $(x, y)$ where $x \in\{0,1, \cdots, N-1\}$ and $y \in\{0,1, \cdots, M-1\}$. The membrane potential $u_{x, y}$ of the neuron $n_{x, y}$ is defined as

$$
\begin{gathered}
u_{x, y}(t)=u_{r e s t}+\eta\left(t-t_{x, y}^{*}\right)+\beta_{x, y} \\
+\xi \times \sum_{n_{x^{\prime}, y^{\prime}} \in B(x, y ; r)} o_{x^{\prime}, y^{\prime}}(t)
\end{gathered}
$$

where $u_{\text {rest }}, t_{x, y}^{*}, \beta_{x, y}, \xi$, and $B(x, y ; r)$ denote the resting potential, the last firing time, the external input, the coupling weight, and the set of connected neurons, respectively. A set of connected neurons is defined as

$$
\begin{gathered}
B(x, y ; r)=\left\{n_{x^{\prime}, y^{\prime}} \mid\left(x^{\prime}, y^{\prime}\right) \neq(x, y)\right. \\
\max \left(\left|x-x^{\prime}\right|,\left|y-y^{\prime}\right|\right) \leq r \\
\left.0 \leq x^{\prime} \leq N-1,0 \leq y^{\prime} \leq M-1\right\}
\end{gathered}
$$

The function $o_{x^{\prime}, y^{\prime}}$ is the output from the connected neuron $n_{x^{\prime}, y^{\prime}}$ and is defined as

$$
o_{x^{\prime}, y^{\prime}}(t)=\sum_{t_{x, y}^{*}<t_{x^{\prime}, y^{\prime}}^{(k)}+\Delta \varepsilon<t} \varepsilon\left(t-t_{x^{\prime}, y^{\prime}}^{(k)}-\Delta \varepsilon\right)
$$

where $t_{x^{\prime}, y^{\prime}}^{(k)}$ denotes the $k$ th firing time of the neuron $n_{x^{\prime}, y^{\prime}}$ and $\Delta \varepsilon$ is the time delay of the synaptic connection. The kernel function $\varepsilon$ describes the response of the synaptic connection. The definition of the kernel function $\varepsilon$ is

$$
\varepsilon(s)=\frac{s}{\tau_{\varepsilon}} \exp \left(-\frac{s}{\tau_{\varepsilon}}\right) \Theta(s),
$$

where $\tau_{\varepsilon}$ is the time constant of the synaptic connection.

\subsection{Gradient Field of a Phase Shift}

In this research, to emphasize the synchronized response in one direction, a gradient field of a phase shift is introduced in Eq. (3). The gradient field of a phase shift is defined as,

$$
\phi_{x, y}(\alpha)=D(\cos (\alpha) x+\sin (\alpha) y) \bmod 2 \pi,
$$

where $D$ is the constant gradient and $\alpha$ is the direction of the gradient. Therefore, $\phi_{x, y}$ is the same value in the orthogonal direction to $\alpha$ in a Chaotic-CNN.

\section{Numerical Analysis of Two Coupled Neurons}

As a preliminary numerical experiment, we examined the synchronization property of two coupled neurons corresponding to a $2 \times 1$ Chaotic-CNN. In this case, the neurons $n_{0,0}$ and $n_{1,0}$ were coupled. In the numerical simulation, the parameter values of the Chaotic-SRM were set as follows: $u_{\text {rest }}=-70 \mathrm{mv}, \theta=-35 \mathrm{mv}$, $\eta_{0}=55, \tau_{\eta}=10, \omega_{\eta_{0}}=\frac{0.75}{2 \pi}, A_{\eta_{0}}=10.9, \tau_{\varepsilon}=1.5$ and $\Delta \varepsilon=0.1$. The external input was chosen as 
$\beta_{0,0}=\beta_{1,0}=52.5$ such that a single neuron exhibits a chaotic spike response.

As an index of synchronization, the crosscorrelation between two spike sequences was analyzed. Let $S_{x, y}$ be the set of the firing times of the neuron $n_{x, y}$. The cross-correlation between $S_{x, y}$ and $S_{x^{\prime}, y^{\prime}}$ with the time shift $\Delta t$ is defined as

$$
\begin{gathered}
C C\left(S_{x, y}, S_{x^{\prime}, y^{\prime}} ; \Delta t\right)=\#\left(\left\{t_{x, y}^{(k)} \in S_{x, y} \mid \exists t_{x^{\prime}, y^{\prime}}^{(l)} \in S_{x^{\prime}, y^{\prime}},\right.\right. \\
\left.\left.\left|t_{x, y}^{(k)}-t_{x^{\prime}, y^{\prime}}^{(l)}-\Delta t\right| \leq \Delta s\right\}\right) / \#\left(S_{x, y}\right)
\end{gathered}
$$

where \# $(X)$ denotes the number of elements of $X$ and $\Delta s$ is the time resolution of coincident firing. In this research, $\Delta s$ was set to $0.5 \mathrm{~ms}$. The auto-correlation was defined as $A C\left(S_{x, y} ; \Delta t\right)=C C\left(S_{x, y}, S_{x, y} ; \Delta t\right)$. In the case of $\Delta t=0$, the cross-correlation $C C\left(S_{x, y}, S_{x^{\prime}, y^{\prime}} ; 0\right)$ corresponds to the ratio of synchronized spikes.

We simulated these two coupled neurons and calculated

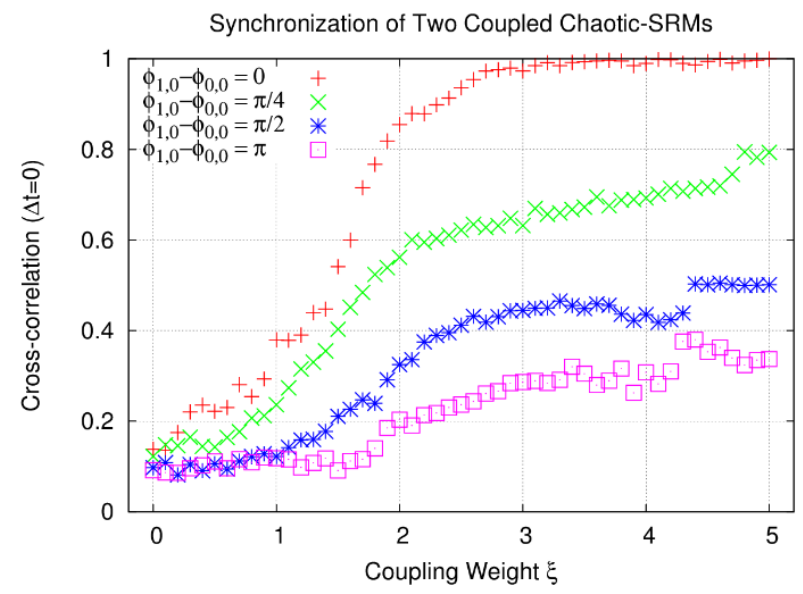

Fig. 1. Cross-correlation vs. coupling weight $\xi$ for two coupled neurons.

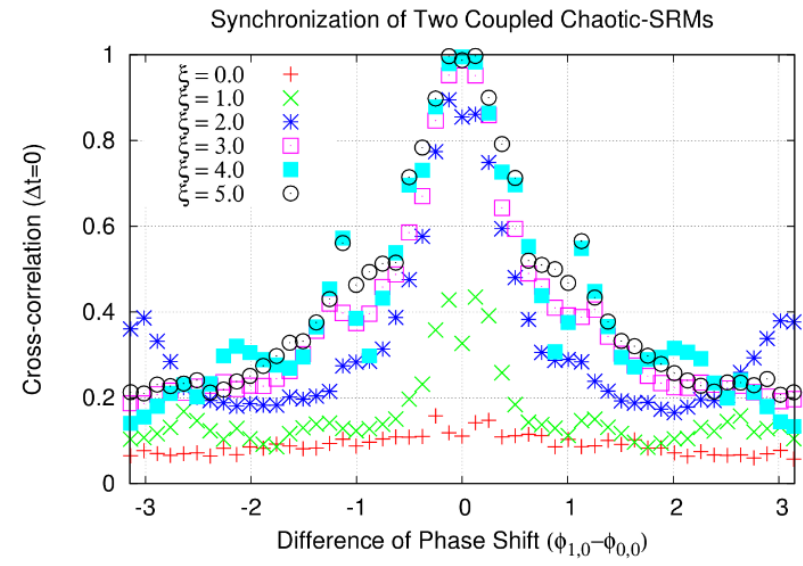

Fig. 2. Cross-correlation vs. phase shift difference $\left(\phi_{1,0}-\right.$ $\left.\phi_{0,0}\right)$ for two coupled neurons. the cross-correlation $C C\left(S_{0,0}, S_{1,0} ; 0\right)$ for the generated spike sequences. Results are shown in Figs. 1 and 2. Fig. 1 shows the dependency of cross-correlation on the coupling weight $\xi$. In the case of equivalent phase shifts for the two neurons $\left(\phi_{0,0}=\phi_{1,0}\right)$, the complete synchronization was such that $\operatorname{CC}\left(S_{0,0}, S_{1,0} ; 0\right) \simeq 1$ was achieved by increasing the coupling weight $\xi$. Fig. 2 shows the dependency of cross-correlation on the phase shift difference $\left(\phi_{1,0}-\phi_{0,0}\right)$. The crosscorrelation exhibits a selective preference for the case where the difference of phase shifts is 0 . In this research, we applied this selective preference of the coupled system to feature linking.

\section{Numerical Experiment}

\subsection{Experimental Model}

In order to examine the feature-linking property using synchronized responses, we constructed a simulation model of a $40 \times 40$ Chaotic-CNN and the motion picture of moving two bars was inputted as a visual stimulus as in physiological experiments investigating a cat's visual cortex ${ }^{1}$. In this research, we also used two motion patterns. One consisted of two bars moving in the same direction (Fig. 3(a)), and the other was two bars moving in opposite directions (Fig. 3(b)).

The image of a bar was represented by a rectangle of $5 \times 12$ pixels. The velocity of each bar was $0.07 \mathrm{pixels} / \mathrm{ms}$. The external input $\beta_{x, y}$ was 52.5 for the neurons inputting the image of the rectangle and 0 for others. The connection parameter $r$ was 2 . The gradient field of the phase shift was set as $D=0.353$ and $\alpha=0$.

\subsection{Cross-Correlation Analysis}

Numerical simulations were performed for spike responses over $1,000 \mathrm{~ms}$. The cross-correlation was then calculated for the neuron $n_{20,10}$ and others. The neuron $n_{20,10}$ was located at the center of the path of bar 1 in Fig. 3.

The cross-correlation for the two bars moving in the same direction (Fig. 3(a)) is shown in Fig. 4(a), which depicts the formation of neural assemblies in which neurons are synchronized. Furthermore, the neural assembly corresponding to bar 1 had high crosscorrelation with the assembly for bar 2. On the other hand, the cross-correlation for the two bars moving in opposite directions (Fig. 3(b)) is shown in Fig. 4(b). Although Fig. 4(b) also shows the formation of a neural assembly, the cross-correlation between the assembly for bar 1 and that for bar 2 was low. 


\section{Discussion}

For the visual stimulus of moving objects, the formation of neural assemblies was observed in the Chaotic-CNN. By cross-correlation analysis, the invoked neural assemblies were found to have high correlation for objects moving in the same direction and low correlation for objects moving in opposite directions.

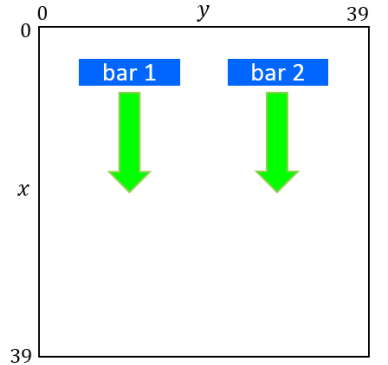

(a) Same direction

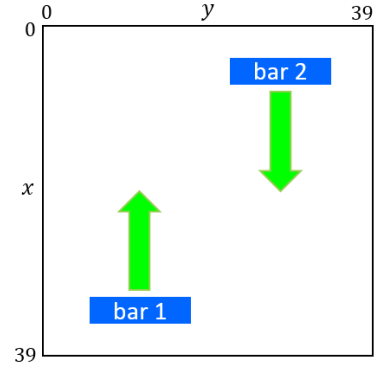

(b) Opposite direction
Fig. 3. Visual stimuli for the numerical experiment. The motion of two bars moving in the same direction (a) and in opposite directions (b).

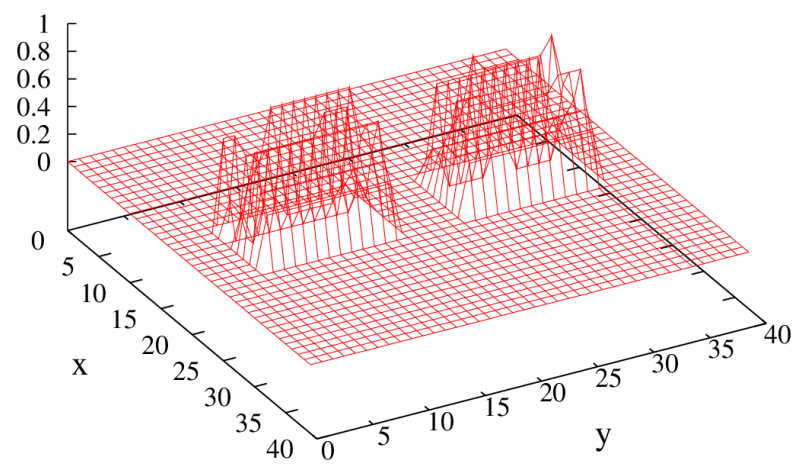

(a) Same direction

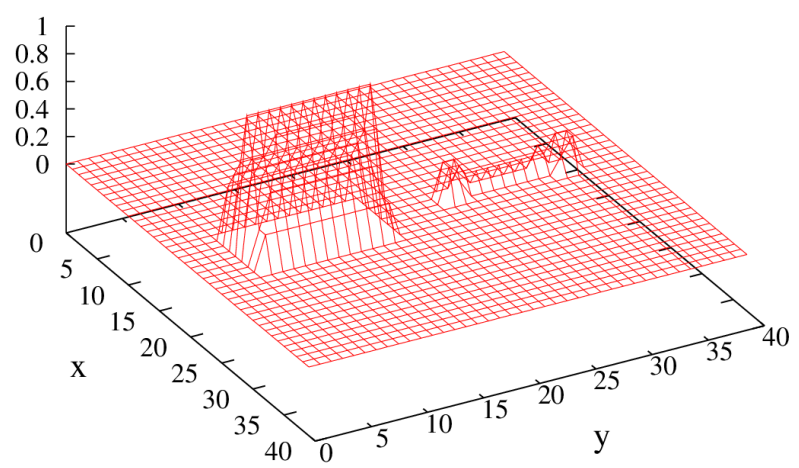

(b) Opposite direction
These results indicate the possibility of feature linking using synchronized spike responses in the Chaotic-CNN in terms of cross-correlation. In our model, this linking property might be achieved by selective preference of the phase shift for the coupled system, as mentioned in Section 3. Analysis of the dynamics of invoked spike sequences along a moving assembly and their application to real images are topics for our future work.

\section{Acknowledgements}

This work was supported by JSPS KAKENHI Grant Number 24650120.

\section{References}

1. Gray, C.M., Koenig, P., Engel, A. K., and Singer, W., Oscillatory responses in cat visual cortex exhibit intercolumnar synchronization which reflects global stimulus properties, Nature, 338, pp. 334-337, 1989.

2. Eckhorn, R., Reitboeck, H.J., Arndt, M., Dicke, P., Feature linking via stimulus-evoked oscillations: experimental results from cat visual cortex and functional implications from a network model, International Joint Conference on Neural Networks, pp. 723-730, 1989.

3. Yamaguchi, A., Okumura, N., Chaki, H., and Wada, M., Chaotic synchronized cluster in the network of spike response neurons, IEICE Tech. Rep., Vol. 99, No. 685, NC99-119, pp. 15-20, Mar. 2000 (in Japanese).

4. Yamaguti, Y., Ishimura, K., and Wada M., Chaotic synchronized assembly in Pulse Coupled Neural Networks, IEICE Tech. Rep., Vol. 101, No. 615, NC2001-98, pp.127-134, Jan. 2002 (in Japanese).

5. Yamaguchi, A., On a chaotic synchronization of one-way coupled two spike response neurons, Fukuoka Institute of Technology Reports of the Computer Science Laboratory, Vol. 24, pp. 1-6, 2013 (in Japanese).

6. Yamaguchi, A., On an information coding using localized synchronization in the two dimensional coupled system of chaotic spike response neurons, Fukuoka Institute of Technology Reports of the Computer Science Laboratory, Vol. 25, pp. 1-6, 2014 (in Japanese).

7. Fujiwara, M., Yamaguchi, A., and Kubo M., Synchronized Response to Grayscale Image Inputs in Chaotic Cellular Neural Network, Journal of Robotics, Networking and Artificial Life, Vol. 2, No. 1, pp. 26-29, 2016.

8. Gerstner, W., and Kistler, W., Spiking Neuron Models: Single Neurons Populations Plasticity, Cambridge University Press, 2002.

9. Lee G., and Farhat, N.H., The Bifurcating Neuron Network 1, Neural Networks, Vol. 14, pp. 115-131, 2001.

Fig. 4. Cross-correlation between neuron $n_{20,10}$ and others when the visual stimuli shown in Fig. 3 were inputted. 